\title{
Energetic particle measurements from the Ulysses/COSPIN/LET instrument obtained during the August/September 2005 events
}

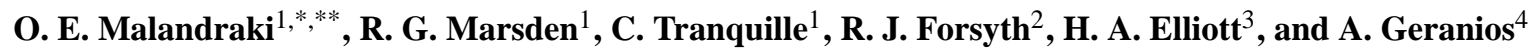 \\ ${ }^{1}$ Research and Scientific Support Department of ESA, ESTEC, Noordwijk, The Netherlands \\ ${ }^{2}$ The Blackett Laboratory, Imperial College of Science and Technology, London, UK \\ ${ }^{3}$ Space Science and Engineering, Southwest Research Institute, San Antonio, TX, USA \\ ${ }^{4}$ Nuclear and Particle Physics Department, University of Athens, Athens, Greece \\ * currently at: The Blackett Laboratory, Imperial College of Science and Technology, London, UK \\ **also at: Institute for Astronomy and Astrophysics, National Observatory of Athens, Athens, Greece
}

Received: 22 December 2006 - Revised: 21 March 2008 - Accepted: 25 March 2008 - Published: 13 May 2008

\begin{abstract}
We report recent observations of energetic particles at energies $1-40 \mathrm{MeV} / \mathrm{n}$ made by the COSPIN/LET instrument onboard the Ulysses spacecraft during the period of intense solar activity in August/September 2005 during the declining phase of solar cycle 23. Ulysses, having started its climb to high southern latitudes for the third time, was located at $\sim 5 \mathrm{AU}$, at a helio-latitude of $\sim 30$ degrees south. It detected the arrival of a solar wind compound stream resulting from the merging of a series of fast halo CMEs ejected from the Sun in late August and early September 2005 and their interaction with the pre-existing pattern of solar wind Stream Interaction Regions (SIRs) in the ambient medium through which they propagated. The heavy ion intensities are observed by COSPIN/LET to remain elevated for at least 20 days following the very intense X17.0/3B solar flare on 7 September and its associated very fast CME (plane of sky projected CME speed $\sim 2400 \mathrm{~km} \mathrm{~s}^{-1}$ ). We carry out an analysis of the composition of the particle increases observed at the location of the spacecraft. Although the composition signatures were predominantly Solar Energetic Particle (SEP)like, after the passage of the compound stream over Ulysses, in association with a characteristic forward and reverse shock pair, the observations showed evidence of an enhanced $\mathrm{He}$ content.
\end{abstract}

Keywords. Interplanetary physics (Energetic particles; Interplanetary magnetic fields; Interplanetary shocks)

Correspondence to: O. E. Malandraki

(omaland@xan.duth.gr)

\section{Introduction}

Ulysses is the first spacecraft ever to fly over the poles of the Sun. Following aphelion passage in June 2004, it began its third climb to high southern heliographic latitudes, now during the declining phase of solar cycle 23. From the end of August through mid-September 2005, a remarkable series of energetic events occurred on the Sun including one of the most intense flares of the solar cycle (see Table 1). The Ulysses spacecraft was near $4.8 \mathrm{AU}$ and about 30 degrees south of the heliographic equator, during this period. This phase of the Ulysses mission provides a unique opportunity to study the effects of solar activity from intermediate latitudes and a heliocentric distance of $\sim 5 \mathrm{AU}$. At this location in the heliosphere, individual transient solar wind disturbances resulting from multiple Coronal Mass Ejections (CMEs) at the Sun tend to coalesce to form larger, more complex structures (e.g. Richardson et al., 2005). If at the same time, the background solar wind is dominated by a more or less regular pattern of fast and slow streams that form Stream Interaction Regions (SIRs) or Corotating Interaction Regions (CIRs) (e.g. Forsyth and Marsch, 1999, and references therein; Gosling et al., 2001; Gosling and Pizzo, 1999), the result is often the formation of so-called compound streams (Burlaga et al., 1986). The focus of this study is the effects of such a compound stream on the populations of energetic particles accelerated by either CME-driven shocks or shocks associated with the stream interactions. In particular, as discussed below, we use composition data to investigate the characteristics of these populations.

Comparison of element abundances in Solar Energetic Particle (SEP) events and CIR-related events at 1 AU shows

Published by Copernicus Publications on behalf of the European Geosciences Union. 
Table 1. Reported Solar Events relevant to the studied particle events from 22 August to 17 September 2005.

\begin{tabular}{|c|c|c|c|c|c|c|c|}
\hline \multicolumn{4}{|c|}{ Flare } & \multicolumn{4}{|c|}{$\mathrm{CME}^{\mathrm{a}}$} \\
\hline Date & X-ray onset & Flare Class & $\mathrm{H} \alpha$ location & NOAA AR & Time $^{\mathrm{b}}$ & CME Width & $V_{\mathrm{CME}}^{\mathrm{c}}$ \\
\hline 22 Aug & $234 / 0044$ & M2.6/SF & S11 W54 & 0798 & $234 / 0131$ & Halo & 1194 \\
\hline 22 Aug & $234 / 1646$ & M5.6/1N & S13 W65 & 0798 & $234 / 1730$ & Halo & 2378 \\
\hline 23 Aug & $235 / 1419$ & $\mathrm{M} 2.7 / \mathrm{SF}$ & S14 W90 & 0798 & $235 / 1454$ & Halo & 1929 \\
\hline 25 Aug & $237 / 0431$ & M6.4/1N & N09 E80 & 0803 & $237 / 0454$ & $146^{\circ}\left(115^{\mathrm{d}}\right)$ & 1327 \\
\hline 28 Aug & $240 / 1017$ & M1.6/SF & N09 E36 & 0803 & $240 / 1056$ & $76^{\circ}(95)$ & 1047 \\
\hline 29 Aug & - & - & - & - & $241 / 1054$ & Halo + & 1600 \\
\hline 31 Aug & - & - & - & - & $243 / 2230$ & Halo + & 1808 \\
\hline 2 Sep & - & - & - & - & $245 / 0030$ & Halo + & 1384 \\
\hline $3 \mathrm{Sep}$ & - & - & - & - & $246 / 0312$ & Halo + & 1672 \\
\hline 5 Sep & - & - & - & - & 248/0948 & Halo + & 2326 \\
\hline $7 \mathrm{Sep}$ & $250 / 1717$ & $\mathbf{X 1 7 . 0 / 3 B}$ & S06 E89 & $0808^{\mathrm{e}}$ & $250 / 1726^{f}$ & $-(105)$ & 2400 \\
\hline 8 Sep & $251 / 2052$ & X5.4/2B & S11 E74 & 0808 & No LASCO, MLSO observations & & \\
\hline 9 Sept & $252 / 0243$ & X1.1 & S12 E68 & 0808 & No LASCO, MLSO observations & & \\
\hline 9 Sep & $252 / 0532$ & M6.2/1F & S13 E71 & 0808 & No LASCO, MLSO observations & & \\
\hline 9 Sep & $252 / 0942$ & X3.6 & S11 E66 & 0808 & No LASCO, MLSO observations & & \\
\hline 9 Sep & $252 / 1913$ & X6.2/2B & S11 E58 & 0808 & $252 / 1948$ & Halo & 2257 \\
\hline 10 Sep & $253 / 0606$ & $\mathrm{M} 3.7 / 2 \mathrm{~N}$ & S11 E51 & 0808 & $253 / 0701$ & $35^{\circ}(149)$ & 747 \\
\hline $10 \mathrm{Sep}$ & $253 / 1634$ & X1.1 & S11 E47 & 0808 & - & - & - \\
\hline $10 \mathrm{Sep}$ & $253 / 1910$ & $\mathrm{M} 4.1 / 1 \mathrm{~N}$ & S12 E45 & 0808 & - & - & - \\
\hline $10 \mathrm{Sep}$ & $253 / 2130$ & $\mathbf{X} 2.1$ & S13 E47 & 0808 & $253 / 2152$ & Halo & 1893 \\
\hline 11 Sep & $254 / 0229$ & M3.4/SF & S12 E42 & 0808 & - & - & - \\
\hline 11 Sep & $254 / 1244$ & M3.0/SF & S13 E42 & 0808 & $254 / 1300$ & Halo & 1922 \\
\hline 12 Sep & $255 / 0837$ & M6.1/2F & S11 E25 & 0808 & $255 / 0912$ & $22^{\circ}(144)$ & 511 \\
\hline $13 \mathrm{Sept}$ & 256/1919 & $\mathrm{X} 1.5 / 2 \mathrm{~B}$ & S09 E10 & 0808 & $256 / 2000$ & Halo & 1866 \\
\hline $13 \mathrm{Sep}$ & $256 / 2315$ & X1.7/1B & S10 E04 & 0808 & - & - & - \\
\hline 14 Sep & $257 / 1005$ & M4.6 & - & 0808 & - & - & - \\
\hline 15 Sep & $258 / 0830$ & X1.1/1B & S10 W15 & 0808 & - & - & - \\
\hline $16 \mathrm{Sep}$ & $259 / 0141$ & M4.4/1B & S13 W26 & 0808 & - & - & - \\
\hline $16 \mathrm{Sep}$ & $259 / 1918$ & $\mathrm{M} 3.5 / 1 \mathrm{~F}$ & S11 W37 & 0808 & - & - & - \\
\hline 17 Sep & $260 / 0558$ & $\mathrm{M} 9.8 / 2 \mathrm{~N}$ & S10 W39 & 0808 & - & - & - \\
\hline
\end{tabular}

a CME classification and parameters extracted from the SOHO/LASCO CME catalogue at http://cdaw.gsfc.nasa.gov/.

b First appearance in the $\mathrm{C} 2$ coronagraph ( $>1.5$ solar radii).

${ }^{\mathrm{c}}$ Plane of sky projected CME speed in $\mathrm{km} \mathrm{s}^{-1}$.

d Position angle measured from solar north in degrees (counterclockwise). + indicates a backside halo CME event.

${ }^{\mathrm{e}}$ Region 0808 is the return of old Region 0798 (as reported in the SEC Preliminary Report and Forecast of Solar Geophysical Data published online at http://sec.noaa.gov.weekly/ by the NOAA/SED and the AFWA).

${ }^{\mathrm{f}}$ Data gap in LASCO, time disruption in the active region near the limb begins, CME observed with the Mk4 K-coronameter at Mauna Loa Solar Observatory (MLSO).

that the latter exhibit significant enrichments of helium and carbon relative to SEP events (Mason and Sanderson, 1999; Fränz et al., 1999; Richardson, 2004, and references therein). Particle composition measurements are important for understanding the origin of the seed particle population accelerated in association with CIRs. It has been suggested that the source is the suprathermal tail of the bulk solar wind distribution (Reames et al., 1991). Another possibility is that CIRs simply re-accelerate a background population of particles remaining from earlier SEP events (e.g. Maclennan et al., 1993; Richardson et al., 1998). A contribution from pick up ions is also a possibility (Gloeckler et al., 1994; Geiss et al., 1995).
However, the contribution of "inner source" pickup ions as a substantial source for the observed enhancement in carbon associated with CIRs is an issue under debate. Recent results based on charge states of CIR-event ions $(\sim 0.5 \mathrm{MeV} / \mathrm{n})$ have shown that "inner source" pick up ions do not appear to be major contributors to the source material for CIRs even at 1 AU (Möbius et al., 2002; Mazur et al., 2002).

Hofer et al. (2003a, b) investigated the origin of the energetic particle populations during and after the second socalled Fast Latitude Scan (that occurred from 27 November 2000 to 13 October 2001) using elemental composition measurements from the Ulysses/COSPIN/LET. They classified 
the energetic particle populations during this period as being mixed between solar energetic particles (major component) and particles accelerated at SIRs or CIRs. CIRs are recurrent SIRs, i.e. compression regions that have a characteristic time difference of $\sim 26$ days as viewed from Ulysses (e.g. Forsyth and Marsch, 1999, and references therein). In the present work, we present recent unique energetic particle measurements in the $1-40 \mathrm{MeV} / \mathrm{n}$ range obtained by the COSPIN/LET instrument onboard Ulysses during the August/September 2005 period of intense solar activity, observed during the declining phase of the solar cycle 23. Furthermore, we carry out a composition analysis which allows distinction between SEPs accelerated in transient events associated with CMEs and particles accelerated at SIRs.

\section{Instrumentation}

The particle data used in this study are from the Low Energy Telescope (LET) onboard the Ulysses spacecraft. LET is one of the five telescopes in the Cosmic Ray and Solar Particle Investigation (COSPIN). The COSPIN/LET instrument records the fluxes and the composition of solar energetic particles low energy cosmic ray nuclei from hydrogen up to iron over a range of energies from $\sim 1 \mathrm{MeV} /$ to $50 \mathrm{MeV} / \mathrm{n}$ using a four-element solid state detector telescope surrounded by an anti-coincidence shield (Simpson et al., 1992). The measurements are made using the double $\mathrm{dE} / \mathrm{dX}$ vs. E technique. The magnetic field observations presented here were made with the Ulysses Magnetometer (Balogh et al., 1992), whilst the solar wind measurements were made with the ion sensor of the Ulysses Solar Wind Plasma experiment (Bame et al., 1992).

\section{Observations and data analysis}

Ulysses data analyzed in this paper cover the period from mid-August to the end of October 2005. Ulysses had begun its third orbit and its ascent to high southern latitudes and during this period was located at $\sim 30$ degrees south of the solar equator, at a heliocentric radial distance of $\sim 4.8 \mathrm{AU}$ from the Sun and traveled in heliolongitude from 160 degrees east to 126 degrees west of the Sun-Earth line.

\subsection{Heliospheric structure and solar activity}

Corotating Interaction Regions are regions of compressed plasma formed at the leading edges of corotating high-speed solar wind streams originating in coronal holes as they interact with the preceding slow solar wind (Balogh et al., 1999). They are particularly prominent features of the solar wind during the declining and minimum phases of the 11year solar cycle. At low heliographic latitudes, such CIRs are typically bounded by forward and reverse waves on their leading and trailing edges, respectively, that steepen into
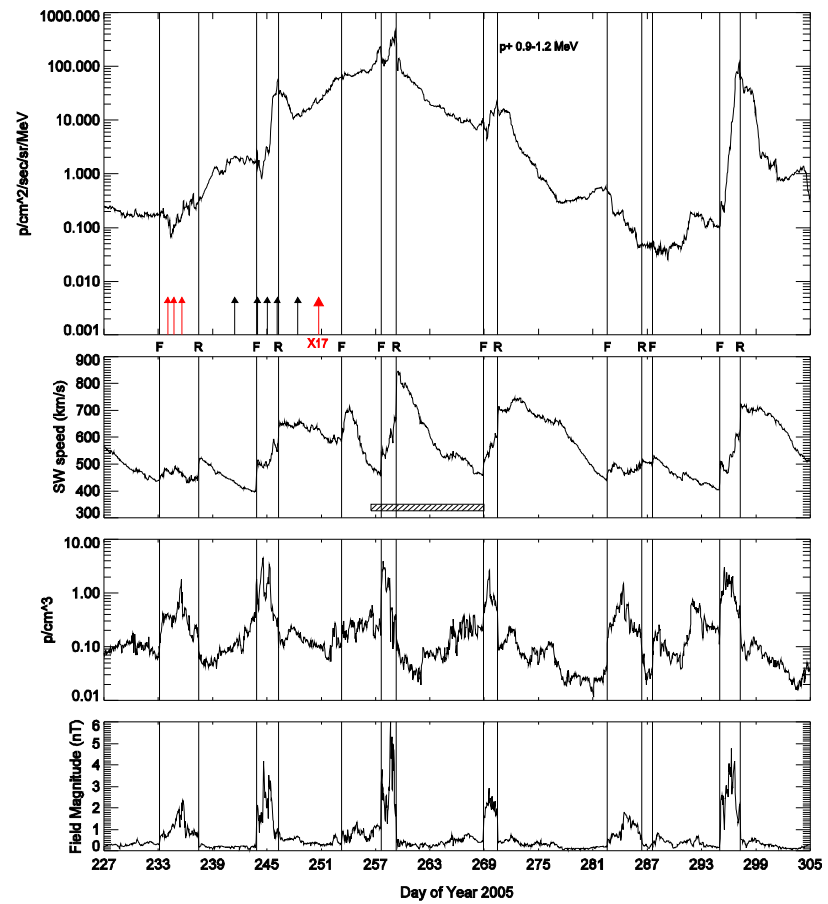

Fig. 1. Overview of the $0.9-1.2 \mathrm{MeV}$ energetic proton intensities as measured by the Ulysses COSPIN/LET instrument, solar wind velocity, plasma ion density and IMF magnitude from 15 August till the end of October 2005 (DOY 227-305). The hatched horizontal bar in the second panel denotes a period with ICME characteristics. Upward pointing red and black vertical arrows indicate solar events that occurred during this period (see Table 1).

shocks at heliocentric distances beyond 1 AU (e.g. Forsyth and Gosling, 2001).

Figure 1 shows from top to bottom an overview of the energetic proton profile in the energy interval $0.9-1.2 \mathrm{MeV}$ as measured by the COSPIN/LET instrument onboard Ulysses, as well as solar wind velocity, density and Interplanetary Magnetic Field (IMF) magnitude observations in the period from 15 August till the end of October 2005 (Days of Year (DOY) 227-305). The solid vertical lines in Fig. 1 correspond to the passage of Forward (F) and Reverse (R) shocks/waves during this period. The Forward shocks are distinguished by an abrupt increase in density, temperature, flow speed, magnetic field strength and total pressure. The Reverse shocks are distinguished by an abrupt decrease in density, temperature, magnetic field strength and total pressure and an increase in flow speed.

The occurrence of stable sources of high speed solar wind streams (i.e. coronal holes) are expected in the descending phase of the solar cycle. As noted above, stable streams of fast solar wind interacting with slow solar wind streams can lead to the formation of compression regions, SIRs or CIRs. During Ulysses' first orbit, in June 1992 while traveling south at $\sim 13^{\circ} \mathrm{S}$ Ulysses encountered a stable high-speed solar 


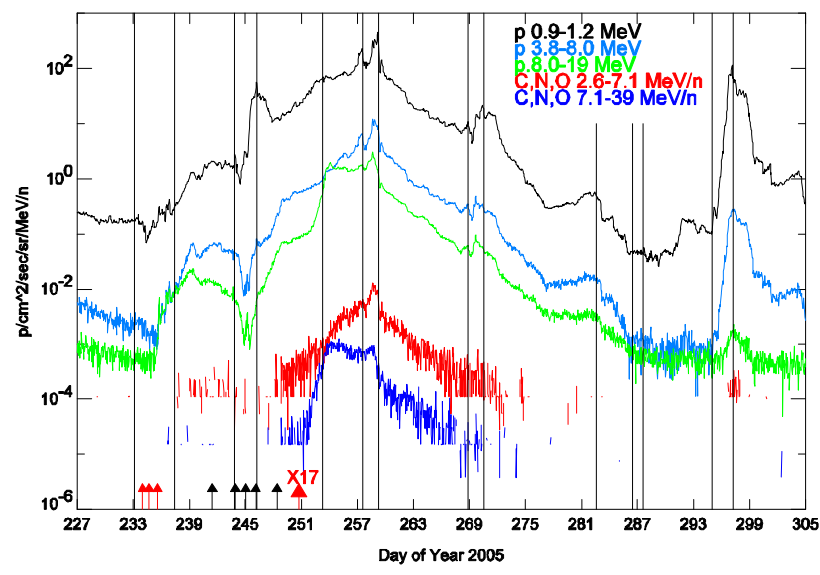

Fig. 2. From top to bottom: Overview of the $0.9-1.2 \mathrm{MeV}, 3.8-$ 8.0 MeV and $8.0-19.0 \mathrm{MeV}$ energetic proton intensities and the heavy ion intensities in the 2.6-7.1 MeV/n and 7.1-39 MeV/n energy intervals for the charge group $\mathrm{C}, \mathrm{N}, \mathrm{O}$ as measured by the Ulysses COSPIN/LET instrument during the period presented in Fig. 1.

wind stream recurring for about 14 rotations through June 1993 at $\sim 34^{\circ} \mathrm{S}$ (Bame et al., 1993). McComas et al. (2006) examined solar wind observations from Ulysses' third solar orbit and concluded that transient disturbances (ICMEs) continued to be observed and that a regular stable stream structure of the kind seen in the first orbit was not recurrently observed at least below $\sim 37^{\circ} \mathrm{S}$. Note that Ulysses finally remained immersed in the southern polar coronal hole highspeed solar wind flow at $39^{\circ} \mathrm{S}$. Nevertheless, Fig. 1 shows that during the period presented, one or more SIRs/CIRs were present (see also Lario and Roelof, 2007). Magnetic field compression regions were clearly observed. The principal stream interaction region passed over the spacecraft around DOY 244 (F-shock on DOY 243 and R-shock on DOY 246). Later occurrences were around DOY 270 (Fshock on DOY 268 and R-shock on DOY 270) and 296 (Fshock on DOY 295 and R-shock on DOY 297). Therefore, these 3 sequences were separated by $\sim 26$ days and constitute a short-lived sequence of CIR events. Weaker SIRs are observed around DOY 235 and 285. A structure with unusually high solar wind speed $(\sim 850 \mathrm{~km} / \mathrm{s})$ and unusually high magnetic field magnitude was observed by Ulysses around DOY 258. As discussed later in Sect. 3.2, this structure has characteristics of interplanetary counterpart of a $\mathrm{CME}$ (i.e. ICME). Forward and Reverse shocks were also observed within the structure, suggesting that the SIRs that passed the spacecraft around DOY 235 and 285 were most likely originated by the same solar wind interaction that produced the forward and reverse shocks in front of this structure (F-shock on day 257 and R-shock on day 259). The difference is that now, the recurrent structure interacted with the ICME resulting in the formation of a compound stream.
Table 1 contains an extensive list of the major solar events (M3.0 or greater flares; CMEs faster than $1000 \mathrm{~km} \mathrm{~s}^{-1}$ ) and their characteristics for the period 22 August (DOY 234) to 17 September 2005 (DOY 260). These events are considered as possible sources of energetic particles observed at Ulysses. For completeness, in Table 1 we also associate flares and CMEs (when observed) for each major event. Association between solar flares and CMEs is based upon their temporal proximity, the longitude of the flare site and the direction of propagation of the CME. The many X-class events during this period of isolated but intense solar activity are highlighted using bold-faced type. The solar flares are as reported in the Solar Geophysical Data (http://sgd.ngdc.noaa. gov/sgd/jsp/solarindex.jsp), whereas CME identification and parameters were obtained from the SOHO/LASCO CME catalogue compiled by Yashiro and G. Michalek (available at http://cdaw.gsfc.nasa.gov). The CME on 7 September 2005, for which no LASCO images are available, is identified from the list of CMEs observed with the Mk4 K-coronameter at Mauna Loa Solar Observatory (MLSO). The details of CME identification and measurements by MLSO are available at http://mlso.hao.ucar.edu/cgi-bin/mlso_logs.cgi.

\subsection{Energetic particle observations}

Figure 2 focuses on the energetic particle observations and shows an overview of the energetic proton profiles in three energy intervals in the range $0.9-19 \mathrm{MeV}$ and the heavy ion intensities in the 2.6-7.1 MeV/n and 7.1-39 MeV/n energy intervals for the charge group $\mathrm{C}, \mathrm{N}, \mathrm{O}$ as measured by the COSPIN/LET instrument onboard Ulysses during the same period presented in Fig. 1. The instrument configuration is such that, at quiet times, the COSPIN/LET 8-19 MeV proton channel responds mainly to galactic cosmic rays, but when the level of solar activity increases, it also responds to protons in the nominal energy range $8-19 \mathrm{MeV}$. Protons of this energy are normally only observed by the LET in association with large solar energetic particle events. These high-energy particle observations (green trace in Fig. 2) show that there were basically three large enhancements at Ulysses during this period.

The proton intensities started to increase gradually on day 235. This increase can be attributed to the solar events from active region AR 0798 occurring on days 234 and 235 on the Western Hemisphere of the Sun as seen from the Earth (Table 1). We indicate the occurrence of these solar events by thin upward pointing red vertical arrows in Fig. 2. These events most likely started filling the heliosphere with energetic particles. On day 245 , before the fluxes have returned to background levels, a new event sets in. The likely source for this particle enhancement is one or more of the fast backside halo CMEs observed between days 241 and 248. The black upward pointing vertical arrows in Fig. 2 indicate the occurrence of these events. These backside CMEs have been associated 
with the old active region AR 0798 (ftp://lasco6.nasom.nasa. gov/pub/lasco/status/LASCO_CME_List_2005). An increase with statistically significant heavy ion intensities in the 2.67.1 MeV/n energy interval for the charge group C,N,O (red line in Fig. 2) is also seen in association with this solar activity.

Figure 3 shows the position of Ulysses with respect to the fixed Sun-Earth line, projected onto the solar equatorial plane as viewed from the north (top) and on a plane perpendicular to the solar equator as viewed from the Earth toward the Sun (bottom) at the beginning, middle and after the end of the period of major solar activity. The ideal Archimedes spiral magnetic field lines connecting Ulysses to the Sun are also shown, calculated using the measured solar wind speed at Ulysses on the three days indicated. As shown in Fig. 3, given that Ulysses was separated by almost $180^{\circ}$ in longitude with respect to the Earth's position, it is highly likely that CMEs that impact Ulysses originate on the backside of the Sun and do not affect Earth.

Intermittently during the period denoted by a hatched horizontal bar in the 2nd panel from top in Fig. 1, a characteristic coherent rotation of the magnetic field direction, low variance magnetic field, enhanced $\mathrm{He}^{++} / \mathrm{H}^{+}$density ratios, increased $\mathrm{O}^{7+} / \mathrm{O}^{6+}$ charge-state ratios and high iron charge states are observed (see http://helio2.estec.esa.int/ulysses/). This provides evidence that material exhibiting ICME characteristics is observed by Ulysses and strongly suggests that during this period, Ulysses detected a system of solar wind transient flows that resulted from the interaction and merging of the fast backside halo CMEs ejected from the Sun from 29 August (DOY 241) till 5 September (DOY 248) 2005 (Table 1). The arrival time of this system of ICME material at the spacecraft is consistent with the times of ejection of the backside halo CMEs at the Sun. As noted in Sect. 1, these ICMEs should merge by the time they reach $5 \mathrm{AU}$. Interaction and coalescence of the transient flows with preexisting stream interaction regions in the ambient medium through which they propagate can lead to the formation of a compound stream. Forward and Reverse shocks are usually observed at various points during the passage of such compound streams over spacecraft at such heliocentric distances (e.g. Burlaga et al., 1986). As discussed in Sect. 3.1, this is also the case here.

When AR 0798 re-appeared on the east limb of the Sun (as viewed from the Earth) on 7 September (DOY 250) it was numbered AR 0808. At $\sim 17: 17$ UT on this day it produced one of the largest flares of the solar cycle 23 , a very intense $\mathrm{X} 17.2 / 3 \mathrm{~B}$ solar flare which had an associated fast east limb CME (plane of sky projected CME speed $\sim 2400 \mathrm{~km} \mathrm{~s}^{-1}$ ). As shown in Fig. 2, in response to this high level of solar activity an additional rise in the $8-19 \mathrm{MeV}$ particle intensities is observed at $\sim 08: 00$ UT on 9 September (DOY 252) superposed upon the already enhanced proton intensities. Associated increases in the low-energy proton intensities and the 2.6-7.1 MeV/n C,N,O group are not observed, however,
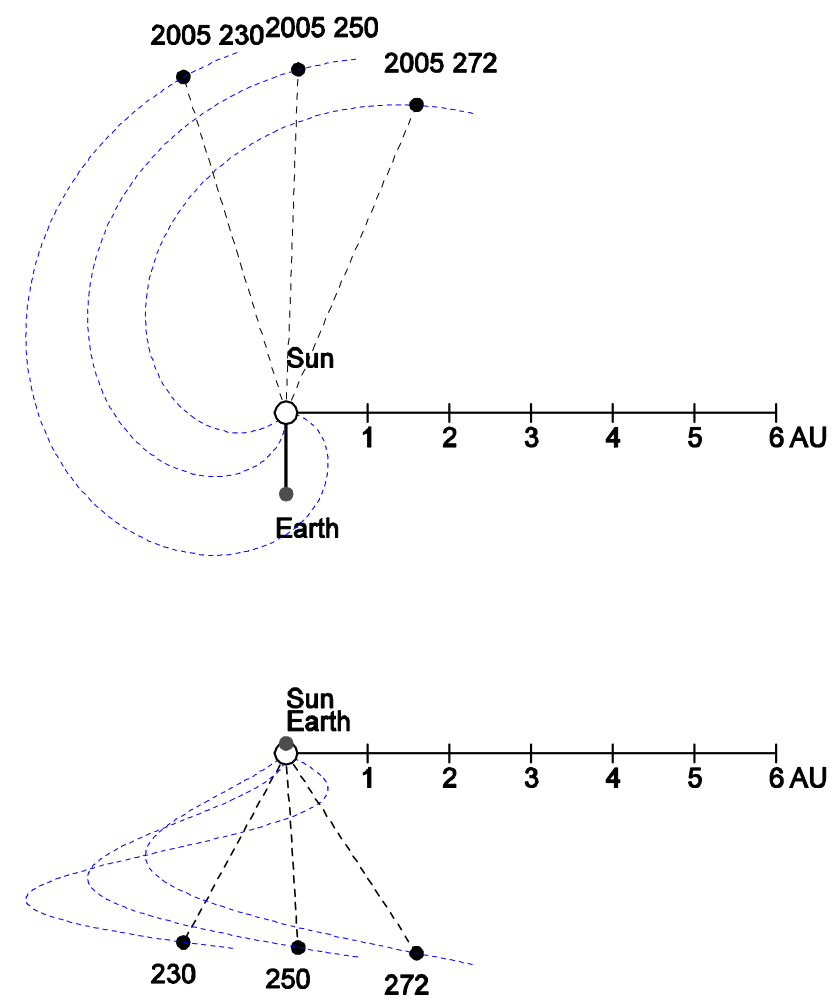

Fig. 3. The position of Ulysses on DOY 230, 250 and 272 of 2005 with respect to the fixed Sun-Earth line, projected (a) on the solar equatorial plane as viewed from the north (top), and (b) on a plane perpendicular to the solar equator as viewed from the Earth toward the Sun (bottom) is shown. Also shown as dashed blue lines are the ideal Archimedes spiral magnetic field lines connecting Ulysses to the solar surface, calculated using the measured solar wind speed.

most likely due to the high pre-event ambient intensities that mask the onset of the event at these channels. The associated onset is more clearly observed in the heavy ion intensities in the 7.1-39 MeV/n energy range for the charge group $\mathrm{C}, \mathrm{N}, \mathrm{O}$ (blue line) which start to rise gradually at $\sim 20: 00 \mathrm{UT}$ on 8 September (DOY 251) with the same slope as the corresponding profile of the $8-19 \mathrm{MeV}$ protons. It is noteworthy that only the X17 solar flare on 7 September produced increases in the C,N,O intensities in the 7.1-39 MeV/n energy range. This is most likely the result of the flare intensity and/or a better magnetic connectivity between Ulysses and the Sun during this period. Using the solar wind velocity of $600 \mathrm{~km} / \mathrm{s}^{-1}$ measured by the Ulysses/SWOOPS experiment at the time of the event the Parker spiral magnetic field line connecting Ulysses to the Sun, is computed to be $\sim 8.86 \mathrm{AU}$ long. Assuming the first arriving particles were accelerated near the Sun in association with the solar events on the 7 September, the earliest expected onset time for directly arriving particles propagating from the Sun along the field using the velocity of the highest energy ions contributing to this channel would be $\sim 4.3 \mathrm{~h}$ after the events. Comparison 

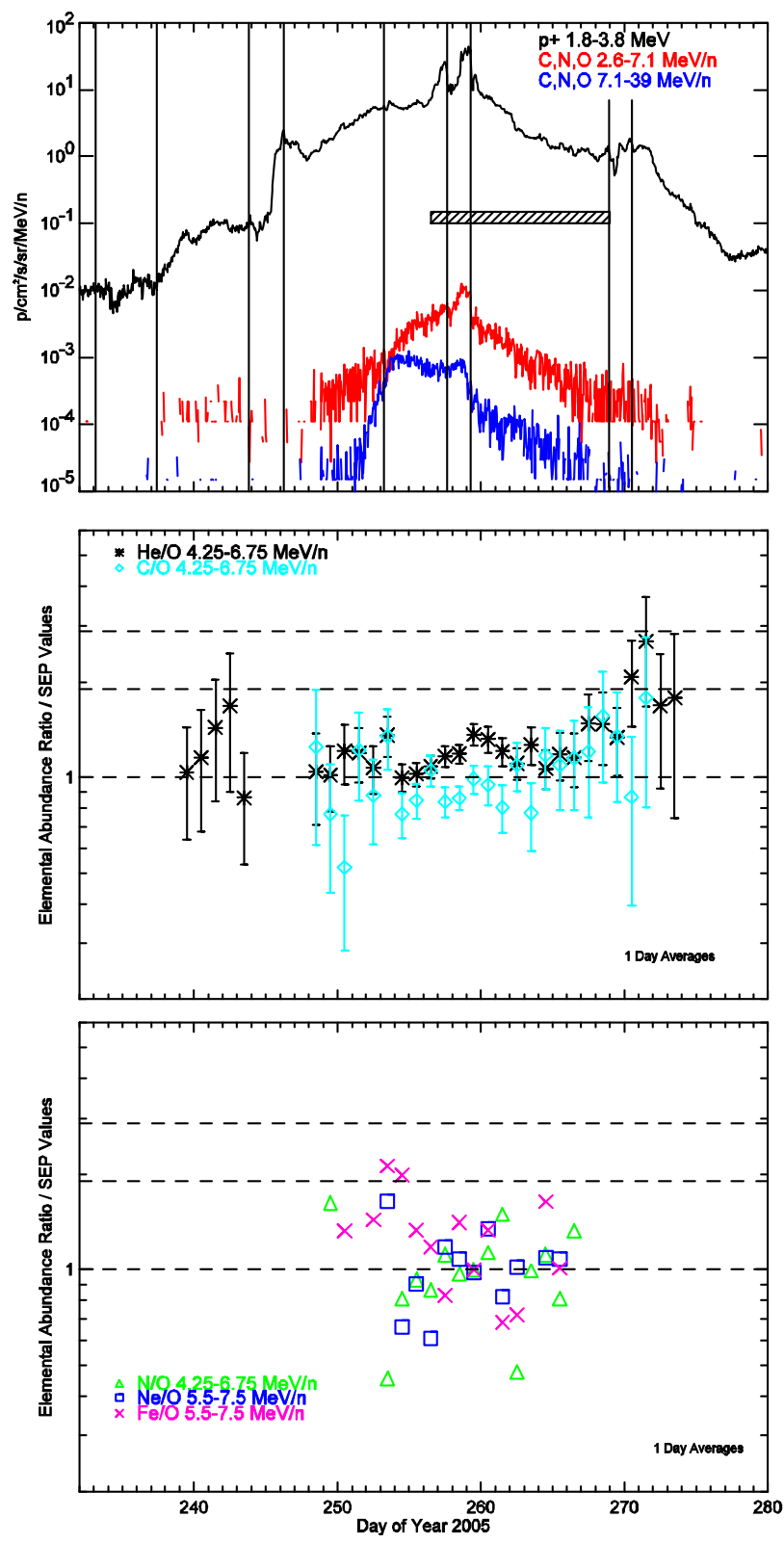

Fig. 4a. (Top panel) The three traces from top show $1.8-3.8 \mathrm{MeV}$ proton intensity and the heavy ion intensities in the $2.6-7.1 \mathrm{MeV} / \mathrm{n}$ and 7.1-39 MeV/n energy intervals for the charge group C,N,O as measured by the COSPIN/LET for the period DOY 232-280. (Middle and bottom panels) Heavy ion abundance ratios (1-day averages) in the energy range $4.25 \mathrm{MeV} / \mathrm{n}$ to $7.5 \mathrm{MeV} / \mathrm{n}$ for helium, carbon, nitrogen, neon and iron with respect to oxygen, all normalized to the SEP composition given by Mason and Sanderson (1999).

with observations shows that the onset at Ulysses is considerably delayed. In this very disturbed period it is more than likely that the actual field lines are significantly distorted from ideal Parker spirals. Furthermore, the observed delay may also result from delayed injection of particles onto IMF lines connecting the particle sources (e.g. CME-driven shocks) to Ulysses.

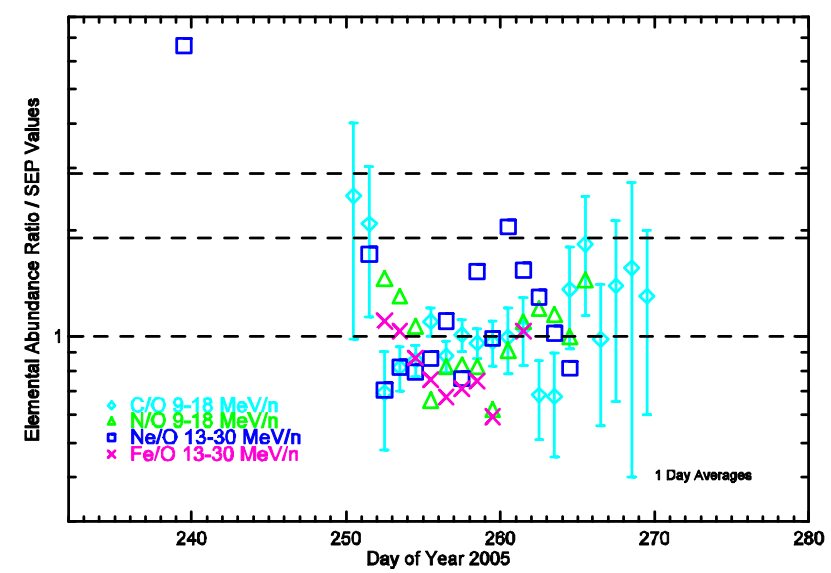

Fig. 4b. Daily averaged elemental abundance ratios $\mathrm{C} / \mathrm{O}$ and $\mathrm{N} / \mathrm{O}$ for the energy range $9-18 \mathrm{MeV}$ and $\mathrm{Ne} / \mathrm{O}$ and $\mathrm{Fe} / \mathrm{O}$ in the energy interval 13-30 MeV/n also normalized to the reference composition given by Mason and Sanderson (1999).

The $8-19 \mathrm{MeV}$ proton intensity (green line in Fig. 2) and the intensity of heavy ions in the 7.1-39 MeV/n energy interval for the C,N,O charge group (blue line in Fig. 2) are observed to exhibit a plateau after they reach a maximum on day 253, while the lower energy heavy ion intensities are observed to still increase gradually. After the passage of the Reverse Shock over the spacecraft on day 259 the particle intensities at all energy channels exhibit a smooth decay. From 7-18 September, (days 250-261) 2005 during its passage on the visible disk of the Sun, AR 0808 produced 26 M-class and $10 \mathrm{X}$-class flares. (Table 1 showing only the flares above M3). This sustained and intense solar activity contributed to the build-up and maintenance at high levels of the particle fluxes at Ulysses.

\subsubsection{Composition analysis}

We now examine the elemental composition of the energetic particle fluxes recorded by the COSPIN/LET instrument onboard Ulysses in association with the August/September 2005 events. The elemental abundances of heavier elements with respect to oxygen can be used to distinguish between SEPs and particle populations associated with SIRs or CIRs (Mason and Sanderson, 1999). For the analysis we divide the measured elemental abundance ratios by a reference value for SEPs. In this analysis we use the reference values as reported by Mason and Sanderson (1999), which refer to Mazur et al. (1993), for both selected energy ranges, i.e. (4.25-7.5) MeV/n and (9.0-30.0) MeV/n. According to Mazur et al. (1993), the reference value for $\mathrm{He} / \mathrm{O}$ is nearly the same in the energy interval $0.6-1.0 \mathrm{MeV} / \mathrm{n}$ and $\sim 4.9-$ $22.5 \mathrm{MeV} / \mathrm{n}$. As reported by Mason and Sanderson (1999), the largest CIR to SEP ratio is obtained for He/O (2.9). Furthermore, the $\mathrm{C} / \mathrm{O}$ ratio given by these authors (1.9) could 
also be used to distinguish between CIR and SEP-related populations. Since there is no large separation of the reference values for the other elements, their elemental abundance ratios are less optimal than $\mathrm{He} / \mathrm{O}$ and $\mathrm{C} / \mathrm{O}$ to identify the type of population.

In the upper panel of Fig. 4a, the 40-min averaged proton intensity in the energy interval $1.8-3.8 \mathrm{MeV} / \mathrm{n}$ and hourly averages of the C,N,O ion intensities in the $2.6-7.1 \mathrm{MeV} / \mathrm{n}$ and 7.1-39 MeV/n energy intervals are shown from top to bottom as measured by the COSPIN/LET for the period 20 August-7 October 2005 (DOY 232-280). The solid vertical lines in this panel mark the time of passage of Forward and Reverse shocks/waves at the Ulysses spacecraft. The hatched horizontal bar denotes the CME/CIR combination observed by Ulysses during this period. In the middle and bottom panels of Fig. 4a, we show the daily averaged elemental abundance ratios for helium, carbon, nitrogen, neon and iron with respect to oxygen for the energy range $4.25 \mathrm{MeV} / \mathrm{n}$ to $7.5 \mathrm{MeV} / \mathrm{n}$ all normalized to the corresponding SEP reference values. Figure $4 \mathrm{~b}$ shows the daily averaged elemental abundance ratios $\mathrm{C} / \mathrm{O}$ and $\mathrm{N} / \mathrm{O}$ for the energy range $9-18 \mathrm{MeV}$ and $\mathrm{Ne} / \mathrm{O}$ and $\mathrm{Fe} / \mathrm{O}$ in the energy interval $13-30 \mathrm{MeV} / \mathrm{n}$ also normalized to the reference composition given by Mason and Sanderson (1999). Statistically significant data available for each element are presented in Fig. 4 (data for each element are shown for which errors are smaller than the elemental abundance ratio value). In order to aid a visual comparison horizontal lines have been drawn at unity, representing the reference SEP value for all elements and at 2.9 and 1.9 marking the reference CIR to SEP value for $\mathrm{He} / \mathrm{O}$ and $\mathrm{C} / \mathrm{O}$ respectively. The plotted error bars for $\mathrm{He}$ and $\mathrm{C}$ take the statistical error and the error of the reference values into account. Data with good statistics for particles in the higher energy range are more limited (Fig. 4b). The normalized elemental abundance ratios plotted in Fig. 4 show that the majority of the particle populations recorded during this period have an SEP elemental composition signature i.e. in Fig. 4 the majority of points lie within the error bars close to a measured-to-SEP ratio at unity for all elements in the energy ranges presented. However, well into the decay phase of the particle event on days 270 and 271, although a quite large scatter in the values making up the daily averages is observed, the normalized $\mathrm{He} / \mathrm{O}$ ratio is apparently enhanced, close to the reference CIR/SEP value. The $(\mathrm{C} / \mathrm{O}) / \mathrm{SEP}$ ratio in both energy ranges shown does not exhibit a corresponding enhancement but, within the error bars, is close to unity throughout the period presented. On days 268 and 270, Ulysses observed a Forward-Reverse shock pair associated with a recurrent CIR (Fig. 1).

\section{Discussion and conclusions}

We report on unique observations obtained from the COSPIN/LET instrument onboard the Ulysses spacecraft at an intermediate latitude of $30^{\circ} \mathrm{S}$ and $\sim 5 \mathrm{AU}$ heliocentric distance during the recent August-September 2005 period of intense solar activity which occurred during the declining phase of the solar cycle 23. Ulysses observes the arrival of a compound stream resulting from the merging of individual transient flows generated by a series of solar events in late August and early September 2005 and their interaction with the pre-existing pattern of stream interaction regions in the ambient medium. A delayed particle onset more clearly observed in the heavy ion intensities in the $7.1-39 \mathrm{MeV} / \mathrm{n}$ energy range for the charge group C,N,O is detected following the very intense X17.0/3B solar flare on 7 September and its associated very fast east limb CME. A possible explanation of the observed delay is a delayed injection of particles onto IMF lines connecting the particle sources to Ulysses. The energetic particle intensities remained elevated at Ulysses for at least 20 days following this intense solar activity.

In our analysis, we have derived the daily averaged elemental abundance ratios based on measurements from the COSPIN/LET instrument. The elemental composition signatures are used to investigate the characteristics of the particle increases observed at the location of the spacecraft. During the CME/CIR combination observed by Ulysses in September 2005 the composition analysis identifies the energetic particles, within the statistical errors, as an SEP population and therefore transient related. Previous elemental abundance analyses at low latitudes with Ulysses (e.g. Hofer et al., 2003a) have shown that while solar energetic particles dominated in the cases studied, there were also indications for particle acceleration at compression regions in a few instances.

As shown in Fig. 4a an enhancement in the He/O ratio is observed after the passage of the compound stream trailing edge over the spacecraft. No corresponding increase in the $\mathrm{C} / \mathrm{O}$ ratio is observed. The He enhancement follows a characteristic recurrent Forward and Reverse shock pair that was observed in the plasma and magnetic field measurements at Ulysses. The He data recorded in this time interval is apparently influenced by this compression region and shows evidence of reverse shock acceleration (Marsden et al., 1993; Richardson et al., 2004). The observation of a more strongly enhanced helium abundance of the reverse shock particles relative to SEP abundances than particles detected at the forward shock is in agreement with current results in CIR research and literature (e.g. Richardson, 2004, and references therein) according to which more efficient particle acceleration occurs in the vicinity of the CIR Reverse shock. We believe that the most plausible origin for the enhanced $\mathrm{He}$ content observed in our paper in association with the CIR is CIR-accelerated pick up helium originating as interstellar neutrals (Simnett et al., 1995). The fact that the enhanced $\mathrm{He} / \mathrm{O}$ values do not reach the CIR/SEP value indicates that Ulysses does not observe a pure CIR effect (e.g. Richardson, 2004) and suggests the existence of a background SEP population of He. The non-observation of an enhancement 
in carbon by Ulysses may be due to a lack of "inner source" pickup carbon at that location or suggests that the bulk of the particles observed were dominated by the ambient SEP population. Another possibility is that the CIR reverse shock has simply reaccelerated a preexisting population of SEPs or particles of this species from the solar energetic particle events themselves.

Acknowledgements. We acknowledge the use of the Ulysses Data System (UDS) in the preparation of this paper. The CME catalogue used to identify the solar origin of the SEP events is generated and maintained by NASA and the Catholic University of America in cooperation with the Naval Research Laboratory. SOHO is a project of international cooperation between ESA and NASA. O. E. Malandraki was supported by an ESA Research Fellowship. This paper is prepared in the frame of the PYTHAGORAS II project, granted by the Greek Ministry of Education.

Editor-in-Chief W. Kofman thanks D. Lario and another anonymous referee for their help in evaluating this paper.

\section{References}

Balogh, A., Beek, T. J., Forsyth, R. J., et al.: The magnetic field investigations on the Ulysses mission: Instrumentation and preliminary scientific results, Astron. Astrophys. Suppl. Ser., 92, 221236, 1992.

Balogh, A., Gosling, J. T., Jokipii, J. R., Kallenbach, R., and Kunow, H. (Eds.): Corotating Interaction Regions, Proceedings of an ISSI Workshop, Kluwer Academic Press, Space Sci. Rev., 89, 1-2, 1999.

Bame, S. J., McComas, D. J., Barraclough, B. L., et al.: The Ulysses solar wind plasma experiment, Astron. Astrophys. Suppl. Ser., 92, 237-265, 1992.

Bame, S. J., Goldstein, B. E., Gosling, J. T., et al.: ULYSSES observations of a recurrent high speed solar wind stream and the heliomagnetic streamer belt, Geophys. Res. Lett., 20, 2323-2326, 1993.

Burlaga, L. F., McDonald, F. B., and Schwenn, R.: Formation of a compound stream between $0.85 \mathrm{AU}$ and $6.2 \mathrm{AU}$ and its effects on solar energetic particles and galactic cosmic rays, J. Geophys. Res., 91, 13 331-13 340, 1986.

Forsyth, R. J. and Marsch, E.: Solar origin and interplanetary evolution of stream interfaces, Space Sci. Rev., 89, 7-20, 1999.

Forsyth, R. J. and Gosling, J. T.: Corotating and transient structures in the heliosphere, in: The Heliosphere Near Solar Minimum: The Ulysses perspective, edited by: Balogh, A., Marsden, R. G., and Smith, E. J., pp. 107-166, Springer-Verlag, UK, 2001.

Fränz, M., Keppler, E., Lauth, U., Reuss, M. K., Mason, G. M., and Mazur, J. E.: Energetic particle abundances at CIR shocks, Geophys. Res. Lett., 26, 17-20, 1999.

Geiss, J., Gloeckler, G., Fisk, L. A., and von Steiger, R.: $C^{+}$Pickup ions in the heliosphere and their origin, J. Geophys. Res., 100, 23 373-23 377, 1995.

Gloeckler, G., Geiss, J., Roelof, E. C., Fisk, L. A., Ipavich, F. M., Ogilvie, K. W., Lanzerotti, L. J., von Steiger, R., and Wilken, B.: Acceleration of interstellar pickup ions in the disturbed solar wind observed on Ulysses, J. Geophys. Res., 99(A9), $17637-$ $17644,1994$.
Gosling, J. T. and Pizzo, V. J.: Formation and evolution of Corotating Interaction Regions and their three dimensional structure, in: Corotating Interaction Regions, edited by: Balogh, A., Gosling, J. T., Jokipii, J. R., Kallenbach, R., and Kunow, H., Space Sci. Rev., 89, 21-52, 1999.

Gosling, J. T., McComas, D. J., Skoug, R. M., and Forsyth, R. J.: Stream interaction regions at High Heliographic Latitudes during Ulysses' Second Polar Orbit, Space Science Rev., 97, 189-192, 2001.

Hofer, M. Y., Marsden, R. G., Sanderson, T. R., et al.: From the Sun's south to the north pole - Ulysses COSPIN/LET composition measurements at solar maximum, Ann. Geophys., 21, 13831393, 2003a, http://www.ann-geophys.net/21/1383/2003/.

Hofer, M. Y., Marsden, R. G., Sanderson, T. R., Tranquille, C., and Forsyth, R.: Transition to solar minimum at high latitudes: Energetic particles from corotating interaction regions, Geophys. Res. Lett., 30(19), 8034, doi:10.1029/2004GL020679, 2003 b.

Lario, D. and Roelof, E. C.: Energetic particles during the first and third Ulysses southern high-latitude excursions: Probing global corotating interaction region structure beyond $5 \mathrm{AU}$, J. Geophys. Res., 112, A09107, doi:10.1029/2007JA012414, 2007.

Maclennan, C. G., Lanzerotti, L. J., Gold, R. E., Hawkins III, S. E., and Tappin, S. J.: Composition of interplanetary ions in recurrent events: Measurements by HI-SCALE on Ulysses at 3-5 AU, Proceedings of the 23rd Intern. Cosmic Ray Conf., 3, 330-334, 1993.

Marsden, R. G., Sanderson, T. R., Wenzel, K.-P., and Bame, S. J.: COSPIN LET observations of recurrent energetic particle events during the in-ecliptic phase of the Ulysses mission, Adv. Space Res., 13/6, 95-98, 1993.

Mason, G. M. and Sanderson, T. R.: CIR associated energetic particles in the inner and middle heliosphere, Space Sci. Rev., 89, 77-90, 1999.

Mazur, J. E., Mason, G. M., Klecker, B., et al.: The abundances of hydrogen, oxygen and iron accelerated in large solar particle events, Astrophys. J., 404, 810-817, 1993.

Mazur, J. E., Mason, G. M., and Mewaldt, R. A.: Charge states of energetic particles from corotating interaction regions as constraints on their source, Astrophys. J., 566, 555-561, 2002.

McComas, D. J., Elliott, H. A., Gosling, J. T., and Skoug, R. M.: Ulysses observations of very different heliospheric structure during the declining phase of solar activity cycle 23 , Geophys. Res. Lett., 33, L09102, doi:10.1029/2006GL025915, 2006.

Möbius, E., Morris, D., Popecki, M. A., Klecker, B., Kistler, L. M., and Galvin, A. B.: Charge states of energetic $(\approx 0.5 \mathrm{MeV} / \mathrm{n})$ ions in corotating interaction regions at $1 \mathrm{AU}$ and implications on source populations, Geophys. Res. Lett., 29(2), 1016, doi:10.1029/2001GL013410, 2002.

Reames, D. V., Richardson, I. G., and Barbier, L. M.: On the differences in element abundances of energetic ions from corotating events and from large solar events, Astrophys. J., 382, L43-L46, 1991.

Richardson, I. G., Mazur, J. E., and Mason, G. M.: A comparison of recurrent energetic ion enhancements observed at Ulysses and at 1 AU by IMP-8 and SAMPEX: Ulysses launch until following the first north polar passage, J. Geophys. Res., 103, 2115-2130, 1998.

Richardson, I. G.: Energetic particles and corotating interaction re- 
gions in the solar wind, Space Sci. Rev., 111, 267-376, 2004.

Richardson, J. D., Wang, C., Kasper, J. C., and Liu, Y.: Propagation of the October/November 2003 CMEs through the heliosphere, Geophys. Res. Lett., 32, L03S03, doi:10.1029/2004GL020679, 2005.

Simnett, G. M., Sayle, K. A., and Roelof, E. C.: Differences between the $0.35-1.0 \mathrm{MeV} /$ nucleon $\mathrm{H} / \mathrm{He}$ ratio in solar and corotating events at high heliolatitude, Geophys. Res. Lett., 22(23), 3365-3368, 1995.
Simpson, J. A., Anglin, J. D., Balogh, A., et al.: The ULYSSES Cosmic Ray and Solar Particle Investigation, Astron. Astrophys. Suppl. Series, 92(2), 365-399, 1992. 